\title{
Cell growth, cell-cycle progress, and antibody production in hybridoma cells cultivated under mild hypothermic conditions
}

\begin{abstract}
The effect of mild hypothermic (32 degrees C) conditions on cell growth, cell-cycle progress, and antibody production of hybridoma C2E7 cells was investigated in the present study. The growth of hybridoma cells was slower during the mild hypothermic condition compared to that at 37 degrees $\mathrm{C}$; this led to about $10 \%$ decrease in maximum viable cell density and volumetric antibody productivity. However, under mild hypothermic growth conditions, the culture viability was substantially improved and the specific antibody productivity was enhanced compared to that at 37 degrees $\mathrm{C}$. The average specific productivity for the entire batch culture at 32 degrees $\mathrm{C}$ is about $5 \%$ higher than that at 37 degrees $\mathrm{C}$. Cell-cycle analysis data showed that there was no growth arrestment during the mild hypothermic growth of hybridoma cells. The G1-phase cells were increased, while the S-phase cells were decreased gradually as the culture time progressed. Further analysis showed that the specific antibody productivity of hybridoma cells was correlated to the fraction of S-phase cells.
\end{abstract}

Keyword: Mild hypothermic condition; Hybridoma; Antibody production; Cell-cycle; Cell growth 